\title{
A Study on an Advanced Lithium-ion Battery System for EVs
}

\author{
Hideaki Horie*, Takaaki Abe*, Takuya Kinoshita*, and Yoshio Shimoida*
}

This paper presents an extensive study concerning a lithium-ion battery system for constructing high-performance power source systems intended to make advanced environmental vehicles a practical reality. Battery performance must be predicted and designed with higher accuracy in order to achieve performance attributes suitable for such power source systems. For example, more quantitative approaches for improving battery power output are needed that are based on a thorough understanding of the fundamental processes which take place in a battery. In line with these perspectives, we constructed a simulation model of electrode reactions and charge transport processes and used it to examine the effects of different factors on battery performance.

This approach is considered to be promising for the construction of a high-performance battery system for EV application. Higher battery performance can be expected from optimization of the electrode parameters. With regard to specific power in particular, the present study examined the possibility of improving battery power output during a short duration.

This paper describes how the concept of short-duration power output might be derived from the electrode characteristics and discusses its potential effects on the overall battery system. It also presents the results of simulations that examined the battery system from the standpoint of thermal behavior.

\section{Keywords: Lithium-ion Battery, EV Battery, Power Characteristics, Short-duration Power Output, Thermal Design}

\section{INTRODUCTION}

There are strong needs today for the development and widespread use of high-performance environmental vehicles owing to concerns about the tightening of energy supplies and global warming resulting from the continued increase in massive energy consumption. Electrical vehicles (EVs) and hybrid electric vehicles (HEVs) have long topped the list of promising environmental vehicles, and R\&D activities for these vehicles have been under way for many years.

A feasibility study was initiated on lithium-ion batteries in 1990, focusing on their performance attributes as a battery technology for constructing a future power supply system for environmental vehicles. The results of preliminary studies conducted at that time indicated that lithium-ion batteries, which were just beginning to appear commercially then, were also very promising for the development of high-performance environmental vehicles. That finding convinced us that

* Next Generation battery development group

Nissan Motor Co., Ltd., 1 Natsusima-cho Yokosuka-shi

Kanagawa 237-8523, Japan

Phone: 81-46-867-5192

Fax: 81-46-867-3611 these batteries had the intrinsic potential to radically transform vehicle power sources. Vigorous full-scale efforts to research and develop lithium-ion batteries for environmental vehicle use were launched in 1992. The first result of those efforts was a cylindrical lithium-ion battery for EV use that was developed in 1995. In the latter half of the 1990s, well over $100 \mathrm{EVs}$ were built with that battery and introduced in both Japan and the U.S. Subsequently, with the consistent aim of improving lithium-ion battery performance, we proposed new concepts for high-performance power supply systems that adopted lithium-ion batteries. Additionally, approaches were pursued for applying them to various types of environmental vehicles [1-14] [17].

The following discussion concerns the efforts made to improve the power output of lithium-ion batteries. For application to $\mathrm{HEVs}$, it is inevitably necessary to increase the battery power. Until around the mid1990s, it had long been firmly believed in the worldwide electrochemical community that lithium-ion batteries were intrinsically incapable of achieving high specific power. The reason was related to the necessity of using an organic solvent with lithium, because if water were used as the electrolyte, the water itself would decompose. Since the ion conduction rate in an organic solvent is 
vastly lower than in water, it would be impossible to obtain a large current from a lithium-ion battery in a short period of time. However, we had fundamental doubts about the validity of that view. Through our analytical studies, we negated the conventional wisdom about the inferior power characteristic of lithium-ion batteries and also showed that, on the contrary, these batteries had the potential for attaining exceptionally high specific power. We were the first researchers in the world to advocate that potential and to substantiate it experimentally as well [18-24].

When considering the application of batteries to various kinds of environmental vehicles, it is necessary to optimize the required battery capacity and power output from the standpoint of the performance of each vehicle type. From around the middle of the 1990s, we have long argued on the basis of our analyses and experimental substantiation that lithium-ion batteries can be adapted to a wide range of vehicle applications from HEVs to plug-in hybrids and even EVs, by taking an innovative approach to the electrode design so as to obtain the power characteristics required by each vehicle type. Power characteristics need to be analyzed from the standpoints of the reactions of the active materials used in the positive and negative electrode assemblies, electron conductivity and ion transport.

Based on these viewpoints, the relationship between the maximum specific power and specific energy of batteries was analyzed numerically. Typical results are shown in Fig. 1. For the sake of comparison, the physical parameters of the materials themselves were kept constant in the calculations. As is well known, EVs and the various types of HEVs differ in terms of their power source and vehicle system, so the relationship between optimum power and energy capacity differs from one vehicle to another. Lithium-ion batteries can achieve a good balance between power output and energy capacity over a wide range of operating regions based on the design of the electrode structure. Our research has reached a level where we can analyze and predict battery performance with good quantitative accuracy.

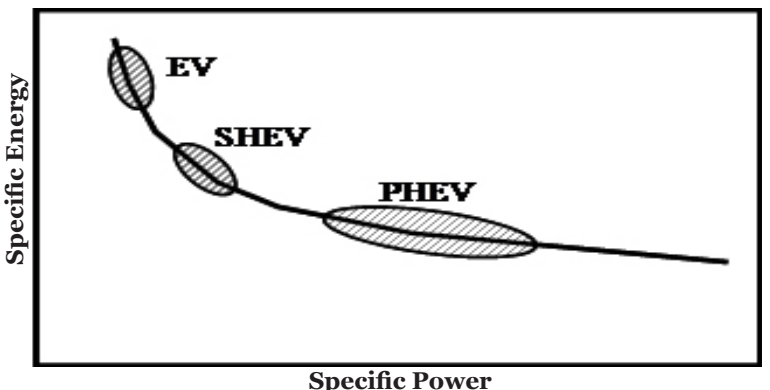

Figure 1: Specific energy vs. specific power
This paper presents a study of approaches for further improving the performance of lithium-ion batteries, specifically for EV application, and also discusses battery system characteristics from the dual perspectives of power and thermal performance.

\section{POWER CHARACTERISTICS OF LITHIUM-ION BATTERIES FOR EV USE}

\subsection{Basic Battery Properties}

As indicated above, there are two basic indices for expressing battery properties: specific energy and specific power. The former index indicates how much energy a battery can store; the latter index indicates the maximum output a battery is capable of producing. Since the driving range of an EV on a single battery charge depends on the amount of energy the battery can store, it is necessary to increase the energy storage capacity further. However, a heavier battery pack would translate directly into a heavier vehicle. For EVs, it is probably safe to say that the biggest issue of concern is the level of the battery's specific energy.

Naturally, the electric devices used to power advanced environmental vehicles must be able to produce the power needed to satisfy the vehicle's power performance requirements. If a battery can no longer produce ample power with only half its capacity, the vehicle's power performance requirements will not be satisfied. If the maximum power design of a battery is too low, the battery will generate more heat, and the battery temperature after driving might become excessively high. High energy efficiency is needed in order to use the limited amount of energy available with maximum effectiveness, but increasing energy efficiency requires a reduction of internal resistance. In other words, the power output of the battery itself must be increased, which is another reason why the power design of a battery becomes a critical factor.

Energy consumption in a battery can be broadly considered in terms of the portion attributable to battery reactions and energy losses accompanying electron or ion transport. As these components become smaller, energy dissipation inside a battery decreases. In other words, internal resistance is reduced and voltage drops across the battery terminals during operation are avoided. With regard to the electrode structure, reducing the thickness of the active material layers lowers the resistance to the passage of lithium ions and electrons through the layers. However, in terms of specific energy, that approach would increase the relative proportion of the cell structure accounted for by components not directly involved in energy storage, such as the current collectors, separators and 
excess electrolyte. As a result, the battery's specific energy would decline.

\subsection{Simulation of Battery Reactions}

An important issue here is the question of how to ascertain internal resistance, i.e., energy dissipation, in a battery more accurately. Because the reaction processes in a battery are extremely complex, any attempt to treat them in terms of simple formulas will definitely not result in accurate predictions. With respect to the internal resistance values of a battery itself, it is necessary to probe the fundamental causes of resistance, understand thoroughly the various mechanisms involved, posit valid hypotheses and formulate quantitative equations. Only by following these steps is it possible to predict transport and reaction processes accurately. These processes are mutually dependent on the material properties of the electrode materials, the geometrical parameters of the electrode structure, the ion concentration, electron conductivity and other variables, as well as on external control variables such as the current value. As such, they can only be obtained by solving a set of equations consisting of many related physical quantities. Based on this way of thinking, we constructed a simulation model of battery reactions and charge transport processes, as shown in Fig. 2 [15-16].

This model consists of schemes for calculating two different transport phenomena:

1. lithium-ion diffusion equations for lithiumion transport in active materials and in the electrolyte, and

2. electron conduction equations.

\subsection{Investigation of Short-duration Battery Power Output for EV Application}

The simulation model was used to conduct various investigations aimed at improving the performance of EV batteries. As the first step, the voltage drop characteristic during battery discharging was investigated for the conventional electrode configuration. Figure 3 shows the cell voltage in relation to the depth of discharge (DOD). The $1 \mathrm{C}$ discharge curve indicates that nearly all the capacity can be extracted in the region of a small discharge current, which is not surprising. However, as the discharge current value is increased, the extractable capacity begins to decrease gradually.

EV batteries not only have to provide average power output during steady-speed driving, they also have to produce large power, albeit for a short duration, during vehicle acceleration. Figure 4 shows the cell voltage characteristics in relation to such short-duration power output for the conventional electrode configuration. At a large discharge current value, it suddenly becomes difficult to maintain the voltage above a certain value. Presumably, that is mainly because lithium-ion diffusion cannot follow the surface reactions, which limits the discharge current.

An investigation was made of methods of extending the short duration of a large-current discharge, with the aim of resolving the issue that such discharges cannot be sustained for a long period of time. First, a simulation was run to investigate the influence of the electrode film thickness. Figures 5 and 6 show the discharge curves obtained when the electrode film thickness was reduced to $84 \%$ and $66 \%$ of the present value, respectively. The results in Figure 5 indicate that

${ }^{\circ}$ Equation for lithium-ion transport in active materials

$$
\frac{\partial C_{s}}{\partial t}=D_{S} \nabla_{r}^{2} \cdot C_{S}
$$

${ }^{\circ}$ Equation for lithium-ion transport in electrolyte

$$
\mathcal{E} \frac{\partial C_{\ell}}{\partial t}=\varepsilon D_{\ell} \nabla_{x}^{2} \cdot C_{\ell}+\frac{\left(1-t_{+}\right)}{F} a i_{n}
$$

${ }^{\circ}$ Equation for current transfer in active materials

$$
\sigma_{\mathrm{S}} \nabla_{\mathrm{x}}^{2} \cdot \emptyset_{\mathrm{S}}-a i_{n}=O
$$

${ }^{\circ}$ Equation for current transfter in electrolyte

$\sigma_{\ell} \nabla_{x}^{2} \bullet \varnothing_{\ell}+a i_{n}-\sigma_{\ell} \nabla_{x}^{2} \bullet\left(\ln C_{\ell}\right) \cdot 2 R T(1-t)=O$

$i_{n}=k C_{\ell}^{x a}\left(C_{t}-C_{s}\right)^{x a} C_{s}^{x c}\left\{\exp \left(\frac{x F}{R T} \eta\right)-\exp \left(-\frac{x F}{R T} \eta\right)\right\}$

$\eta=\varnothing_{s}-\varnothing_{\ell}-U(C)$

Figure 2: Simulation model 


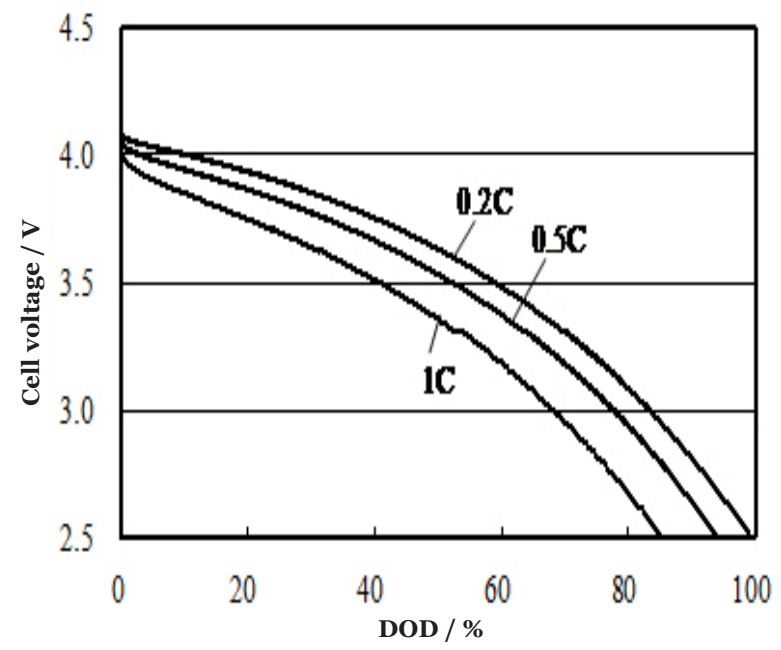

Figure 3: Cell discharge characteristics (conventional electrodes)

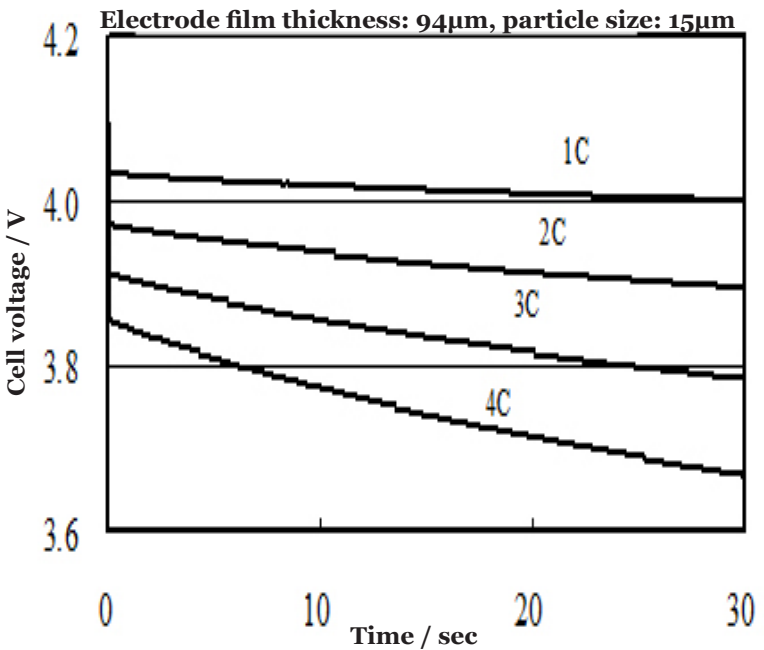

Figure 5: Short-duration large-current discharge characteristics (film thickness reduced to $84 \%$ )

the discharge duration of a $4 \mathrm{C}$ discharge until a level of $3.6 \mathrm{~V}$ was extended by nearly twofold (compared with Fig. 4) when the electrode film thickness was reduced to $84 \%(94 \mu \mathrm{m})$ of the present value. As indicated in Figure 6, the discharge duration was extended by approximately fourfold (compared with Fig. 4) when the film thickness was reduced to $66 \%(74 \mu \mathrm{m})$ of the present value.

An investigation was then made of the influence of the particle size of the electrode active material. Discharge curves are shown in Figures 7 and 8 for two different particle sizes. Looking at the results

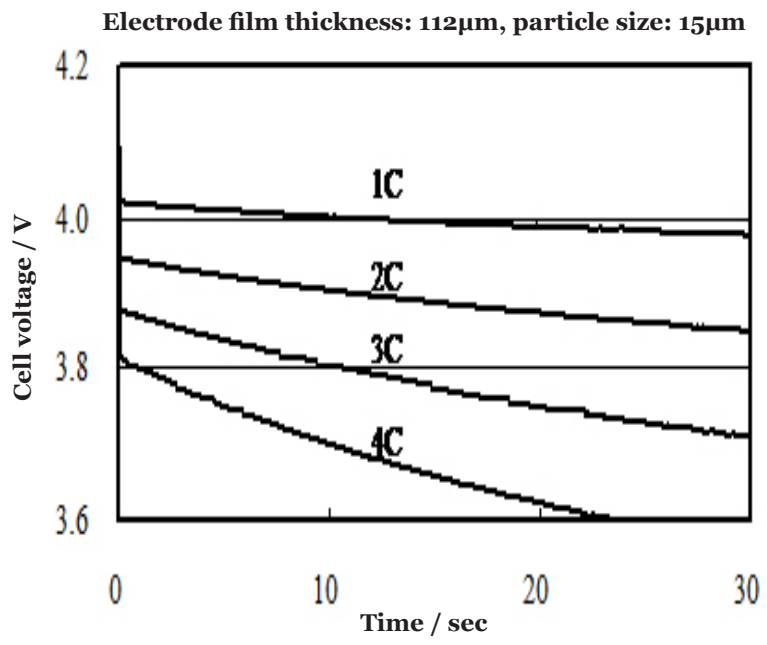

Figure 4: Voltage characteristics of short-duration large-current discharges (conventional electrodes)

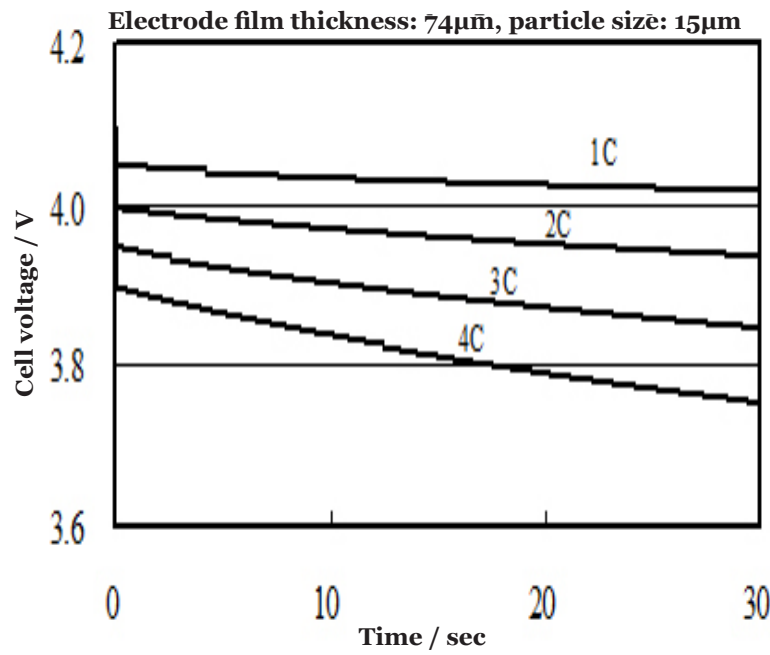

Figure 6: Short-duration large-current discharge characteristics (film thickness reduced to 66\%)

again for a $4 \mathrm{C}$ discharge, it is seen in Figure 8 that the discharge duration was extended by more than threefold (compared with Fig. 4) when the particle size was reduced to $33 \%$ of the present value. That is attributed in part to a reduction of reaction resistance due to the increased reaction area as a result of reducing the particle size. Another reason is thought to be a reduction of ion diffusion resistance due to the shortening of the diffusion distance in the solid layer.

A further investigation was made of the influence of the porosity of the active material. Figures 9 and 10 show the discharge curves when the porosity of the material 
inside the electrodes was increased by $33 \%$ and $67 \%$, respectively. Looking again at a $4 \mathrm{C}$ discharge, it is seen in Figure 10 that the discharge time was extended by approximately twofold (compared with Fig. 4) when the porosity was increased by $67 \%$.

The results of these investigations indicate that the short-duration discharge characteristics of a battery can be changed by modifying the electrode structure.

\section{THERMAL INVESTIGATION OF LITHIUM-ION BATTERY SYSTEM FOR EV USE}

\subsection{Investigation of Short-duration Battery Power Output for EV Application}

The amount of heat generated by the battery increases when large power is demanded for propelling the vehicle. Accordingly, in designing the battery system, it is necessary not only to secure the required power and capacity but also to ensure that the battery system is thermally feasible. Heat generated by a battery includes Joule heat due to internal resistance accompanying charging/discharging processes and the heat of reaction produced by charging/discharging reactions. Such heat generation raises the battery temperature, and continued exposure to high temperatures over a long period of time might accelerate battery degradation. Therefore, it is necessary to keep the battery temperature from rising excessively by applying a suitable cooling system.

\subsection{Temperature Rise of EV Batteries}

A thermal simulation was conducted to examine the thermal behavior of lithium-ion batteries during

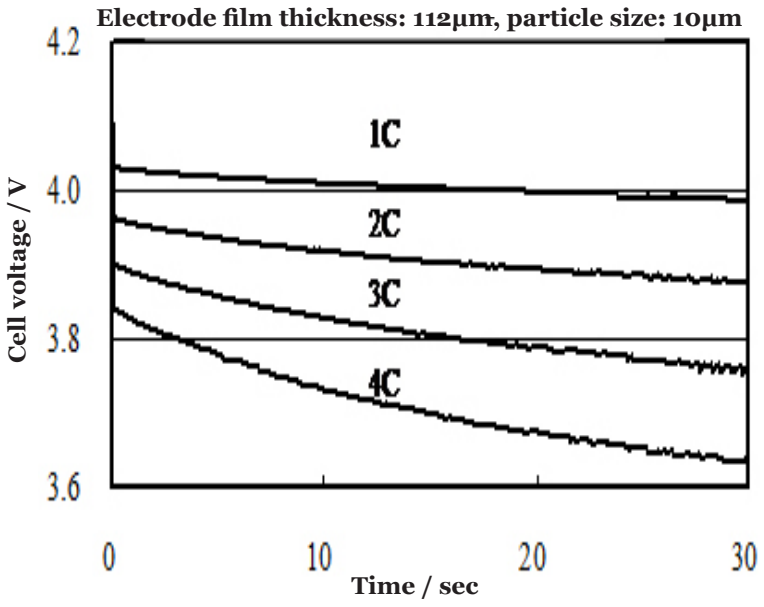

Figure 7: Short-duration large-current discharge characteristics (particle size reduced to 67\%)

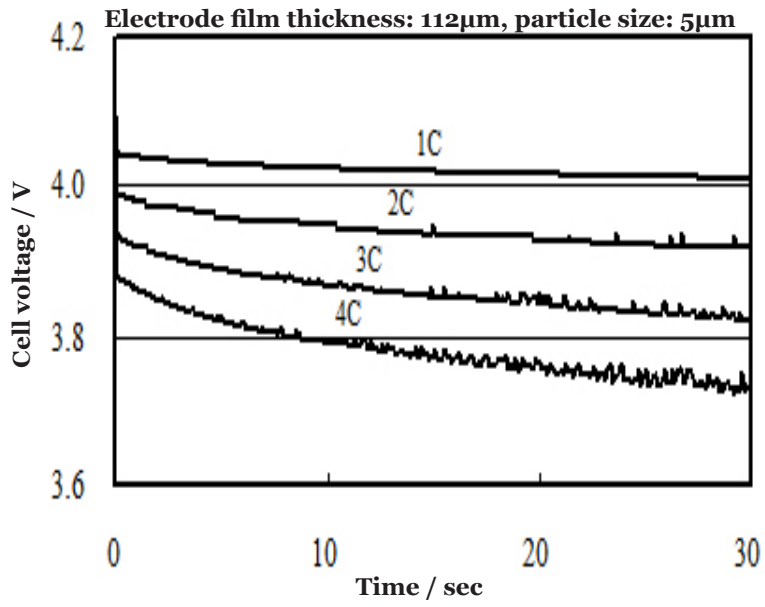

Figure 8: Short-duration large-current discharge characteristics (particle size reduced to 33\%)

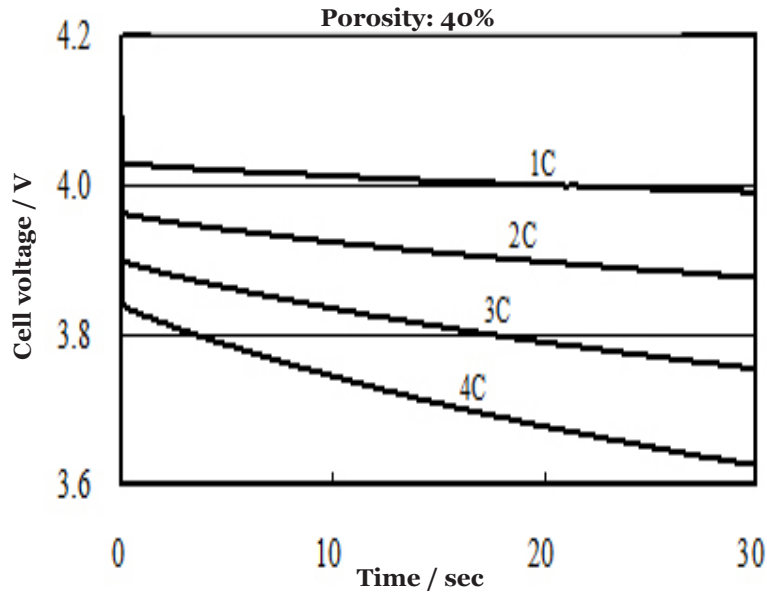

Figure 9: Short-duration large-current discharge characteristics (porosity increased by $33 \%$ )

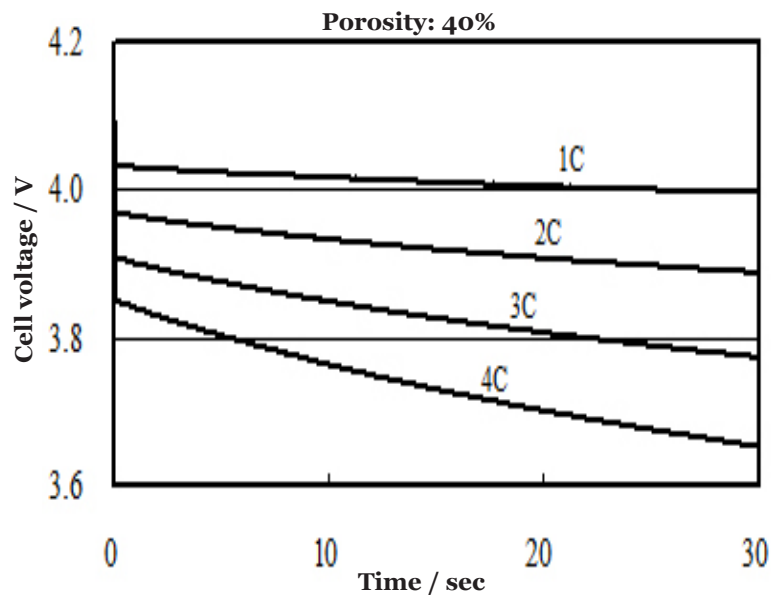

Figure 10: Short-duration large-current discharge characteristics (porosity increased by $67 \%$ )

A Study on an Advanced Lithium-ion Battery System for EVs 
EV operation. The batteries were assumed to be the conventional cylindrical battery module used in the preceding calculations and a laminated battery module that has been the focus of R\&D work since 2000 and has improved short-duration power performance. The temperature rise results are shown in Fig. 11.

The results show a significant difference in the temperature characteristics of the two batteries. Specifically, the temperature rise of the laminated battery module capable of producing a large current for a short duration is effectively suppressed by reducing the electrode film thickness and the particle size of the active material.

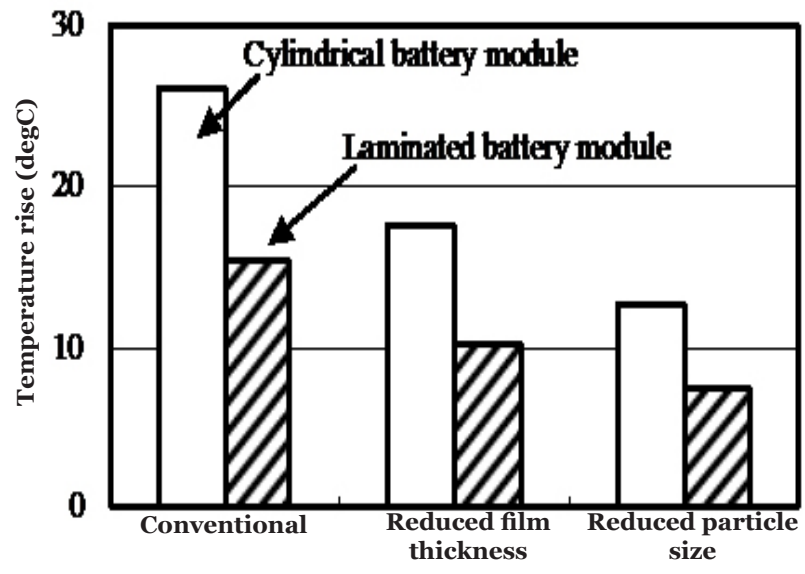

Figure 11: Comparison of temperature rise

\section{CONCLUSION}

A simulation model of electrode reactions and charge transport processes was constructed with the aim of predicting battery performance more accurately in order to reflect that information in the battery system design. The model was used to conduct various investigations, focusing especially on power performance. The results revealed that optimizing the electrode parameters of lithium-ion batteries makes it possible to construct power source systems that match the requirements of a wide variety of environmental vehicles. In the present study, we went another step forward and conducted more in-depth investigations concerning ways of improving EV battery performance in order to achieve lithium-ion batteries that better fit the performance requirements of advanced environmental vehicles.

It was noted that the two major parameters of the fundamental performance required of batteries are (1) specific energy and (2) specific power. With regard to specific power in particular, this study examined not only the maximum rated power but also the possibility of high power output for a short duration and its effects on the battery system. Considering the large difference between the average power output during steady-speed driving and the power needed for vehicle acceleration, it can be posited that a concept which might be called (3) short-duration power output also exists as a third factor of battery performance. Specifically, the results of the present study imply that this short-duration power output can be varied by optimizing the electrode design, making it possible to design battery systems matching different vehicle requirements. Furthermore, in combination with thermal analysis simulations, investigations were also made of battery systems with respect to thermal considerations. The results of this study suggest that lithium-ion battery systems are optimally suited to EV use because the battery performance and vehicle compatibility can be further improved on the basis of the electrode design.

\section{REFERENCES}

[1] H. Horie, et al., Development of a Lithium-ion Battery System for EV Application, Preprints of the Spring

Scientific Lecture Series of JSAE, 961 (1996) (in Japanese).

[2] T. Miyamoto, et al., Advanced Battery System for Electric Vehicle (FEV-II), EVS13 (1996).

[3] H. Horie, et al., Development of a High-power Lithium-ion Battery System for HEV Application, Preprints

of the Spring Scientific Lecture Series of JSAE, 971 (1997) (in Japanese).

[4] N. Hirata, et al., Thermal Management System of Lithium-ion Battery for The NISSAN ALTRA-EV,

EVS-14 (1997).

[5] H. Horie, et al., Development of A high Power Lithium-Ion Battery System for HEV, EVS14 (1997).

[6] T. Kikuchi, et al., Evaluation Tests of Nissan Hybrid Electric Vehicle, EVS14 (1997).

[7] H. Horie, et al., Development of a PHEV Power Supply System using High-power Lithium-ion Batteries,

Preprints of the Autumn Scientific Lecture Series of JSAE, 90, 38633 (1998) (in Japanese).

[8] T. Abe, et al., Development of a Cooling System for High-power Lithium-ion Batteries for $\mathrm{HEV}$ Application,

Preprints of the Spring Scientific Lecture Series of JSAE, (1998) (in Japanese).

[9] S. Kitada, et al., Development of a Parallel HEV System Incorporating a CVT, EVS-15 (1998).

[10] Y. Tanjo, et al., Abstract for the 40th Battery Forum, 2C04 (1999) (in Japanese). 
[11] H. Horie, et al., Development of a High-Power Lithium-ion Battery for Parallel HEVs, EVS16 (1999).

[12] Y. Ohsawa, et al., Abstract for the 41st Battery Forum, 3C15 (2000) (in Japanese).

[13] M. Origuchi, et al., Lithium-ion Battery Application to the Tino Hybrid, EVS17 (2000).

[14] E. Inada, et al., Development of a High-Performance Hybrid Electric Vehicle, "Tino Hybrid", EVS17 (2000).

[15] F. Saito, et al., Verifications of the Battery Adaptation in the Market and Estimation of Battery Life for EV,

HEV, EVS18 (2001).

[16] K. Watanabe, et al., Study of a High-Power Lithiumion Battery, The206th Meeting of The Electrochemical Society, Abs. 441 (2004).

[17] H. Horie, et al., Compact Lithium-ion Battery, JSAE Review, Vol. 58, No. 7 (2004) (in Japanese).

[18] O. Shimamura, et al., Development of a Compact, High-power Lithium-ion Battery System, Transactions of

JSAE, Vol. 36, No. 4, pp. 138-139 (2004) (in Japanese).

[19] H. Horie, et al., Development of Ultra-high Power Lithium-ion Batteries, IMLB-12 (12th International Meeting of Lithium Batteries), Abs.50 (2004).

[20] O. Shimamura, et al., Development of a High Power Compact Lithium-ion Battery System, EVS-21 (2005).

[21] O. Shimamura, et al., Research and Development Work on Lithium-ion Batteries for Environmental Vehicles, EVS-22 (2006).

[22] T. Abe, et al., Research and Development Work on Lithium-ion Battery for Environmental Vehicle, To be presented at the Autumn Scientific Lecture Series of JSAE, Sept. 2006 (in Japanese).

[23] T. Abe, et al., Simulation of a High-Power Lithiumion Battery, The 200th Meeting of The Electrochemical Society, Abs. 133 (2001).

[24] T. Abe, et al., Abstract for the 42nd Battery Forum, 3C05 (2001) (in Japanese)

\section{AUTHORS}

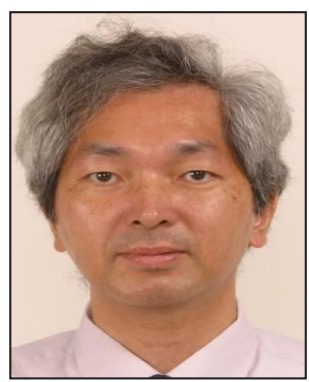

Hideaki Horie, Next Generation battery development group, Nissan Motor Co., Ltd., 1 Natsusima-cho Yokosuka-shi Kanagawa 237-8523, Japan, Phone: 81-46-867-5192, Fax: 81-46-867-3611, Hi-horie@mail. nissan.co.jp.

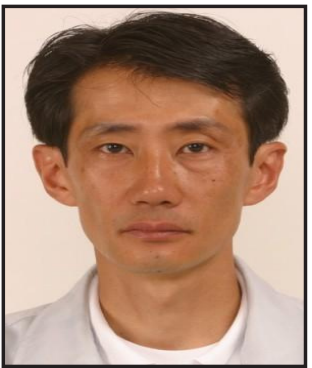

Takaaki Abe, Next Generation battery development group, Nissan Motor Co., Ltd. 1 Natsusima-cho Yokosuka-shi Kanagawa 237-8523, Japan, Phone: 81-46-867-5192, Fax: 81-46-867-3611, takaaki-abe@ mail.nissan.co.jp.

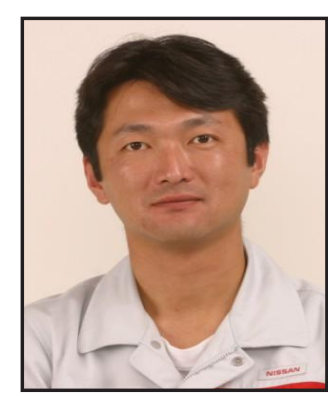

Takuya Kinoshita, Next Generationbatterydevelopment group, Nissan Motor Co., Ltd. 1 Natsusima-cho Yokosuka-shi Kanagawa 237-8523, Japan, Phone: 81-46-867-5192, Fax: +81-46-867-3611, Hi-horie@ mail.nissan.co.jp.

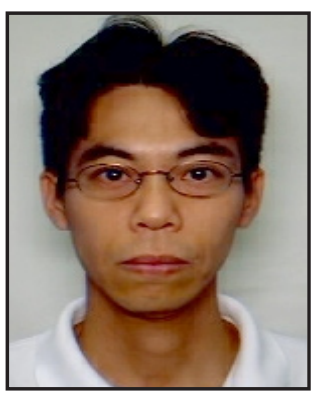

Yoshio Shimoida, Next Generationbatterydevelopment group, Nissan Motor Co., Ltd. 1 Natsusima-cho Yokosuka-shi Kanagawa 237-8523, Japan, Phone: 81-46-867-5192, Fax: 81-46-867-3611, y-shimoida@ mail.nissan.co.jp. 